\title{
Phenotypic variability in Angelman syndrome: comparison among different deletion classes and between deletion and UPD subjects
}

\author{
Monica Castro Varela ${ }^{*},{ }^{1}$ Fernando Kok $^{2}$, Paulo Alberto Otto ${ }^{1}$ and \\ Celia Priszkulnik Koiffmann ${ }^{1}$
}

\begin{abstract}
${ }^{1}$ Centro de Estudos do Genoma Humano, Department of Biology, Institute of Biosciences, University of São Paulo, São Paulo, SP, Brazil; ${ }^{2}$ Child Neurology Service, Department of Neurology, Hospital das Clínicas, University of
\end{abstract} São Paulo School of Medicine, São Paulo, Brazil

Angelman syndrome (AS) can result from either a 15q11-q13 deletion (del), paternal uniparental disomy (UPD), imprinting, or UBE3A mutations. Here, we describe the phenotypic and behavioral variability detected in 49 patients with different classes of deletions and nine patients with UPD. Diagnosis was made by methylation pattern analysis of exon 1 of the SNRPN-SNURF gene and by microsatellite profiling of loci within and outside the $15 q 11-q 13$ region. There were no major phenotypic differences between the two main classes (BP1-BP3; BP2-BP3) of AS deletion patients, except for the absence of vocalization, more prevalent in patients with BP1-BP3 deletions, and for the age of sitting without support, which was lower in patients with BP2-BP3 deletions. Our data suggest that gene deletions (NIPA1, NIPA2, CYF1P1, GCP5) mapped to the region between breakpoints BP1 and BP2 may be involved in the severity of speech impairment, since all BP1-BP3 deletion patients showed complete absence of vocalization, while $38.1 \%$ of the BP2-BP3 deletion patients were able to pronounce syllabic sounds, with doubtful meaning. Compared to UPD patients, deletion patients presented a higher incidence of swallowing disorders $(73.9 \%$ del $\times$ $22.2 \%$ UPD) and hypotonia (73.3\% del $\times 28.57 \%$ UPD). In addition, children with UPD showed better physical growth, fewer or no seizures, a lower incidence of microcephaly, less ataxia and higher cognitive skills. As a consequence of their milder or less typical phenotype, AS may remain undiagnosed, leading to an overall underdiagnosis of the disease.

European Journal of Human Genetics (2004) 12, 987-992. doi:10.1038/sj.ejhg.5201264

Published online 6 October 2004

Keywords: Angelman syndrome; maternal 15q11-q13 deletion; breakpoints; paternal uniparental disomy; phenotypic variability; diagnosis

\section{Introduction}

Angelman syndrome (AS) ${ }^{1}$ comprises developmental delay, severe mental retardation, absent speech, seizures, ataxia, outbursts of laughter, microcephaly, brachycephaly,

*Correspondence: Dr MC Varela, Departamento de Biologia, Instituto de Biociências, USP, Caixa Postal 11.461, CEP: 05422-970, São Paulo, SP, Brazil. Tel: + 55113031 4304; Fax: + 55113091 7553;

E-mail: mcvarela@ib.usp.br or cpkoiffm@ib.usp.br

Received 17 March 2004; revised 11 June 2004; accepted 25 June 2004 macrostomia, and prognathism. Gait is described as widebased, with arms held flexed and upheld at the elbows. AS is caused by the loss of expression of maternal imprinted gene(s) mapped to the chromosome region 15q11-q13.

AS and Prader-Willi syndrome (PWS - neonatal hypotonia, poor sucking, delayed psychomotor development, hyperphagia, obesity, short stature in adolescents and adults, small hands and feet, hypogonadism, mild to moderate mental retardation, temper tantrums, obsessivecompulsive mannerisms) were the first examples in hu- 
mans of genomic imprinting or the differential expression of genetic material, depending on the parent of origin. While AS results from the loss of expression of a maternal gene $(U B E 3 A)$, PWS results from the loss of expression of paternal imprinted genes mapped to the chromosome region $15 \mathrm{q} 11-\mathrm{q} 13$.

Four different mechanisms can lead to the AS phenotype: two-thirds of AS cases have a maternal deletion within 15q11-q13; paternal uniparental disomy of chromosome 15 (UPD15) is detected in 2-3\%; approximately, $2 \%$ have a mutation in the imprinting center and, in about $8 \%$, mutations in the UBE3A gene are found.

The chromosome region $15 \mathrm{q} 11-\mathrm{q} 13$ is meiotically unstable, with an unusual variety of cytogenetic rearrangements, including the AS and PWS deletions, duplications and triplications, inv $\operatorname{dup}(15)$ marker chromosomes, inversions, and balanced or unbalanced translocations. In 95\% of PWS/AS patients with a deletion, two main classes of deletions are found. ${ }^{2-4}$ Class I patients show breakpoints at BP1 (proximal) and BP3 (distal), while Class II patients present breakpoints at BP2 (proximal) and BP3 (distal). The remaining 5\% have the distal breakpoint at BP4. Another breakpoint, BP5, has been reported only in inv dup(15) marker chromosomes ${ }^{5,6}$ and in some cases of interstitial duplications and triplications of chromosome 15q11q13. ${ }^{7-9}$ Recent studies have shown that the deletions in 15q11-q13 are mediated by nonallelic homologous recombination between low-copy repeats (duplicons), which map to the common deletion breakpoint regions. ${ }^{3,4}$

In general, patients with AS resulting from large chromosome deletions appear to be more severely affected than patients belonging to the other genetic classes. Several authors have shown that AS patients with UPD have milder phenotypes than patients with deletions. ${ }^{10-13}$ They pointed out that children with UPD have a better physical growth, frequently with weight above the 75 th centile, fewer or no seizures, less ataxia, and better cognitive skills. Hypopigmentation is more frequent among patients with deletions, since the nonimprinted gene responsible for pigmentation (P) that causes type II oculocutaneous albinism ${ }^{14}$ is located in the distal portion of $15 \mathrm{q} 11-\mathrm{q} 13$.

When compared to deletion AS subjects, patients with imprinting defects are less likely to have microcephaly, hypopigmentation, or seizures, and show better growth, motor milestones, and communication skills. Obesity is relatively common in this group. ${ }^{15}$

Patients with UBE3A mutations have abilities that fall somewhere in between those of the deletion and the UPD group. They frequently have seizures and microcephaly, but hypopigmentation is not detected. ${ }^{16}$ Motor and communication skills are better than in the deletion group. Lossie et $a l^{17}$ pointed out that in this group the frequency of obesity is particularly high as the patients get older.
Herein we report the proximal and distal breakpoints of chromosome segment 15q11-q13 detected in 46 deletion AS patients (13 BP1-BP3 - Class I; 22 BP2-BP3 - Class II; two BP2-BP4 - Class III, one BP2-BP5 - Class IV, eight inconclusive) and describe the phenotypic and behavioral variability detected among 35 patients with different classes of deletions (13 Class I; 22 Class II) and among 49 patients with deletions and nine patients with UPD.

\section{Materials and methods \\ Patients}

Investigation of breakpoints was carried out on 46 AS deletion patients, and phenotypic and behavioral studies on 58 patients ( 34 female and 24 male subjects with ages ranging from 1 year and 3 months to 30 years and 5 months): 49 with a 15q11-q13 deletion and nine with UPD. These patients were diagnosed in our laboratory from July 1996 through July 2003.

Most patients were referred for genetic testing for AS by physicians of the Neurology Department and the Children's Institute of the University of São Paulo School of Medicine and were examined by at least one of the authors, following a standard protocol that included evaluation of physical and behavioral characteristics. Informed consent was obtained for all patients from a legal guardian.

In all, 21 of the patients with a deletion and four with UPD were previously reported in Fridman et $a l^{13}$ and another eight of the nine UPD patients presented here were described in Fridman et al. ${ }^{18}$

\section{Genetic studies}

DNA was extracted from peripheral blood leukocytes by standard procedures. Diagnosis was established by methylation pattern analysis of the PWS/AS region. The DNA was modified by bisulfite treatment, and the SNURF-SNRPN exon 1 amplified by PCR. ${ }^{19}$ A characteristic AS pattern is recognized by the presence of the $221 \mathrm{bp}$ paternal band only (data not shown).

Three markers within the critical region $15 \mathrm{q} 11-\mathrm{q} 13$ (D15S11, D15S113 and GABRB3) ${ }^{20}$ and at least one marker outside this region (D15S984, D15S131, D15S117, D15S115 or CYP19) were studied in patients and their parents, to distinguish between deletion and paternal uniparental disomy (data not shown).

Investigation of the extent of the deletion was performed by microsatellite analysis with markers mapped to the segment 15q11-q14 (D15S11, D15S113, GABRB3, D15S1002, D15S1048, D15S1019, D15S165, D15S1031, D15S1043, D15S1010) in patients and their parents.

Chromosome studies of patients were performed on peripheral blood lymphocytes, using the GTG-banding technique to investigate structural and numerical alterations. 


\section{Statistical analysis}

Absolute frequencies of phenotypic characteristics in subgroups of patients were compared in $2 \times 2$ contingency tables, using Fisher's exact test that generates the exact probabilities corresponding to the null hypothesis of nonassociation. Quantitative measurements and counts were contrasted using Mann-Whitney's nonparametric test.

\section{Results}

All patients presented normal karyotypes. Of 46 AS deletion patients analyzed with D15S541/D15S542 markers, 41 were informative for at least one of the two markers. Overall, in 34.46\% (14/41), a S541/542 deletion was found, indicating that BP1 was the proximal deletion breakpoint. The other $65.54 \%(27 / 41)$ were heterozygous at $S 541 / 542$, indicating that BP2 was the proximal breakpoint. Of 43 informative cases, 40 (93.02\%) presented the distal breakpoint at BP3 (proximal to D15S1048/1019); $4.66 \%(2 / 43)$ at BP4 (between D15S1019 and D15S165), and $2.32 \%(1 / 43)$ at BP5 (between D15S1031 and D15S1010). In summary, we found five breakpoint regions in AS deletion patients: BP1, BP2, BP3, BP4, and BP5. A total of 38 patients presented informative results for proximal and distal breakpoints concomitantly: $34.21 \%$ (13) belonging to Class I (BP1-BP3), 57.9\% (22) to Class II (BP2-BP3), 5.26\% (two) to Class III (BP2-BP4), and 2.63\% (one) to Class IV (BP2-BP5).

Table 1 shows the frequency of the clinical and behavioral findings among patients with different classes of deletions (13 patients with deletions at BP1-BP3, 22 $\mathrm{BP} 2-\mathrm{BP} 3$, two BP2-BP4, one BP2-BP5). Table 2 shows the frequency of the clinical and behavioral characteristics of the 49 deletion and nine UPD patients.

\section{Discussion \\ Comparison of behavioral and clinical findings among AS deletion patients with different classes of deletion}

Our deletion patients had the same breakpoints already described, ${ }^{2-4}$ with the exception of the distal breakpoint BP5, found in a single patient. A distal BP5 breakpoint had previously been detected only in large inv dup(15) chromosomes $^{5,6}$ and in some cases of interstitial duplications and triplications of chromosome $15 q 11-q 13 .^{7-9}$

All deletion patients presented a clinical phenotype typical of AS: developmental delay, severe mental retardation, macrostomia, outbursts of laughter, and ataxic gait. Class II deletion patients showed a better performance than Class I subjects in two developmental areas: sitting without support and vocalization. Sitting without support was achieved, on the average, at 16 months by Class II patients and at 19 months by Class I patients. Vocalization was more articulated in Class II, in which $38.1 \%$ of patients were able to pronounce syllabic sounds of doubtful meaning. Class I patients could only utter unarticulated sounds. No statistical differences could be demonstrated with respect to the age of neck support and independent gait, but Class II patients seemed to have a slightly better motor development (Table 1).

Class I patients also showed a higher incidence of hypotonia $(84.6 \%$ in Class I $\times 61.1 \%$ in Class II), microcephaly $(75 \%$ in Class I $\times 55 \%$ in Class II); seizures (present in 92.3\% Class I $\times 85 \%$ Class II) and lower ability of communication by gestures $(60 \%$ in Class $\mathrm{I} \times 84.6 \%$ in Class II), although no statistical significance could be demonstrated.

The present study was able to establish a correlation between two different major deletion classes and the AS phenotype. Recently, four genes (NIPA1, NIPA2, CYF1P1, GCP5) were mapped to the region between breakpoints BP1 and BP2, but their function is still unknown. ${ }^{21}$ Rainier et $a l^{22}$ reported a dominant negative mutation in the NIPA1 gene in a kindred with autosomal dominant hereditary spastic paraplegia linked to the SPG6 locus. The fact that Class I deletion patients with PWS and AS do not exhibit progressive spastic paraplegia indicates that NIPA1 haploinsufficiency does not cause this disease.

Our data suggest that deletions of the genes mapped to the region between $\mathrm{BP} 1$ and $\mathrm{BP} 2$ might be involved in speech impairment and delayed acquisition of developmental abilities, since all BP1-BP3 deletion patients showed complete absence of vocalization, while $38.1 \%$ of the BP2-BP3 deletion patients were able to pronounce syllabic sounds, and the developmental delay was more severe in AS patients with BP1-BP3 than with BP2-BP3 deletions.

In a recent study, Butler et $a l^{23}$ found that PWS subjects with Class I deletions have a more severe phenotype than those with Class II deletions, including self-injurious behavior, deficits in adaptive behavior (including motor skills), obsessive-compulsive behavior, and difficulties with reading, mathematics skills, and visual-motor integration.

\section{Comparison of behavioral and clinical findings between AS deletion patients and paternal UPD patients}

Our previous report on 21 AS deletion patients and four paternal UPD patients ${ }^{13}$ stated that UPD patients use to be diagnosed later than deletion patients, mainly because the phenotypic and behavioral traits are more subtle in UPD children. Microcephaly and complete absence of speech were more frequent among deletion patients; UPD patients usually walked earlier and had seizures later than deletion patients. In the present study, we also observed an older age at diagnosis for UPD (average 9 years) than for deletion patients (average 5 years and 8 months). This was also 
Table 1 Phenotypic characteristics of AS patients according to deletion class

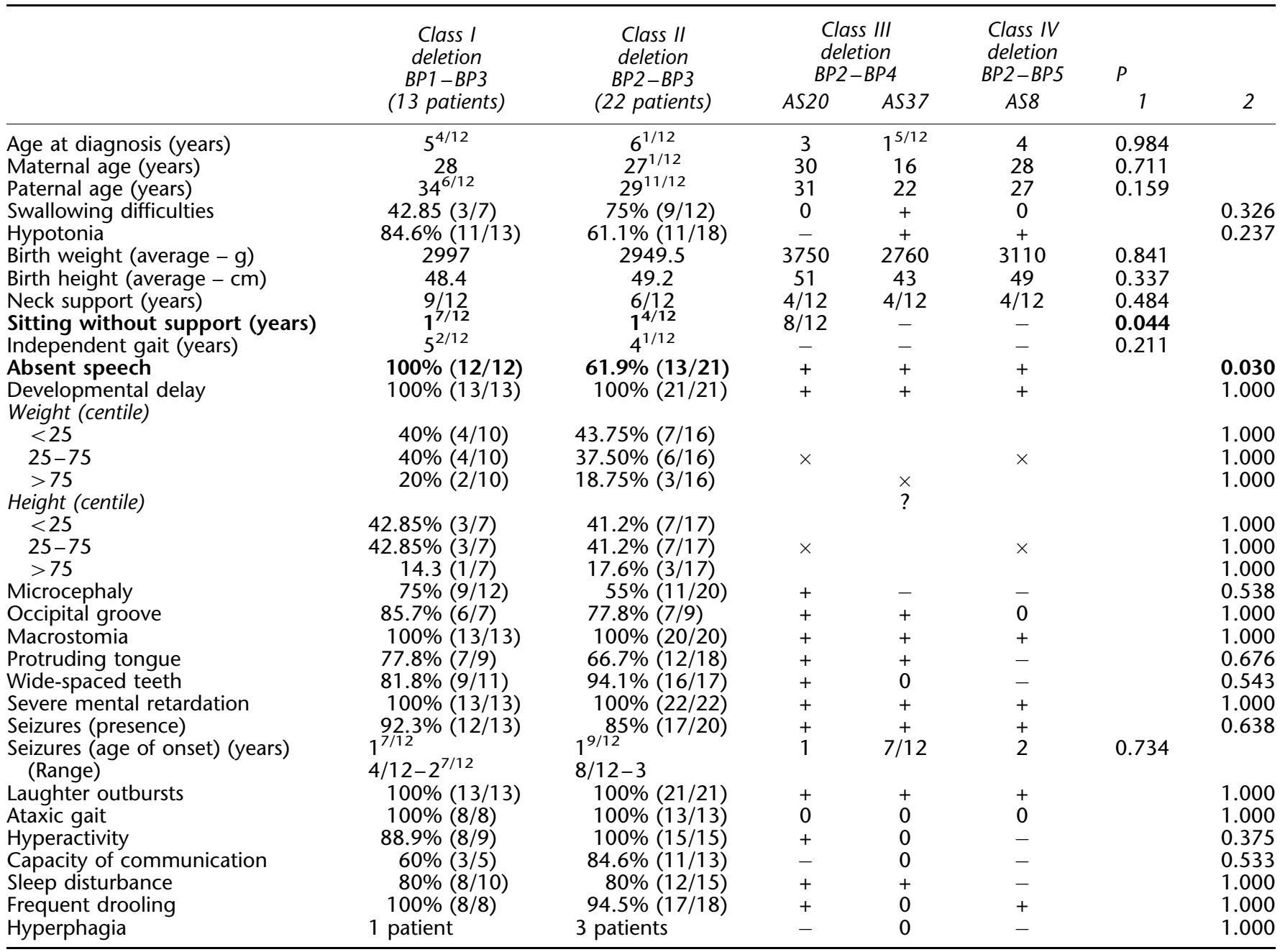

$P$ : Results of statistical tests for differences between Class I and Class II deletion patients.

1: Mann-Whitney's test.

2: Fisher's exact test.

Significant results $(P<0.05)$ are indicated in bold.

reported by Bottani et al, ${ }^{10}$ Gillenssen-Kaesbach et al, ${ }^{11}$ and Smith et al. ${ }^{12}$

We did not detect any significant difference between maternal and paternal ages of UPD and deletion AS patients (Table 2), although the origin of the syndrome in UPD individuals usually depends on nondisjunction events that are associated with increased maternal age. In AS, most paternal UPD15 seem to be postzygotic events. $^{24,18}$

In the present study, in addition to the differences described previously, ${ }^{13}$ deletion patients presented a higher incidence of swallowing disorders $(73.9 \times 22.2 \%)$ and hypotonia $(73.3 \times 28.57 \%)$ than UPD patients.

Other features associated with the clinical diagnosis of AS, such as brachycephaly, occipital groove, macrostomia, wide-spaced teeth, outbursts of laughter, hyperactivity, sleep disturbance and frequent drooling, presented a higher incidence among deletion patients, suggesting that these patients have a more severe and more typical phenotype, although these differences did not reach statistical significance (Table 2). Haploinsufficiency of genes localized in the deleted chromosome segment is probably responsible for the more severe phenotypic and behavioral characteristics of deletion patients, as compared to patients with UPD, imprinting mutations, and UBE3A mutations.

The fact that UPD patients have a milder or less typical phenotype suggests that AS may be underdiagnosed. Based on our study, we recommend that developmental delay, severe mental retardation, speech impairment, happy 
Table 2 Phenotypic characteristics of AS patients with deletions and UPD

\begin{tabular}{|c|c|c|c|c|}
\hline & \multirow[b]{2}{*}{ Deletion (49 patients) } & \multirow[b]{2}{*}{ UPD (9 patients) } & \multicolumn{2}{|c|}{$P$} \\
\hline & & & 1 & 2 \\
\hline \multirow{2}{*}{$\begin{array}{l}\text { Age at diagnosis (years) } \\
\text { (Range) }\end{array}$} & $\begin{array}{c}5^{8 / 12} \\
1^{3 / 12}-30^{5 / 12}\end{array}$ & $\begin{array}{l}9 \\
27 / 12-21\end{array}$ & 0.087 & \\
\hline & 29F; 20M & $5 \mathrm{~F} ; 4 \mathrm{M}$ & & \\
\hline \multirow{2}{*}{$\begin{array}{l}\text { Maternal age (years) } \\
\text { (Range) }\end{array}$} & $27^{8 / 12}$ & $29^{5 / 12}$ & 0.562 & \\
\hline & $16-39$ & $23-40$ & & \\
\hline \multirow{2}{*}{$\begin{array}{l}\text { Paternal age (years) } \\
\text { (Range) }\end{array}$} & $32^{1 / 12}$ & $33^{9 / 12}$ & 0.497 & \\
\hline & $20-52$ & $25-47$ & & \\
\hline Birth weight (average - g) & 2981 & 3328 & 0.055 & \\
\hline & 49 & 48.5 & 0.447 & \\
\hline $\begin{array}{l}\text { Birth height (average }-\mathrm{cm} \text { ) } \\
\text { Swallowing difficulties }\end{array}$ & $73.9 \%(17 / 23)$ & $22.22 \%(2 / 9)$ & & 0.015 \\
\hline Hypotonia & $73.33 \%(33 / 45)$ & $28.57 \%(2 / 7)$ & & 0.031 \\
\hline \multirow{2}{*}{ Neck support (years) } & $7 / 12$ & $4 / 12$ & 0.059 & \\
\hline & $1^{5 / 12}$ & 1 & 0.184 & \\
\hline Independent qait (years) & $4^{4 / 12}$ & 3 & 0.136 & \\
\hline Absent speech & $91.5 \%(43 / 47)$ & $66.6 \%(6 / 9)$ & & 0.074 \\
\hline \multirow{2}{*}{$\begin{array}{l}\text { Developmental delay } \\
\text { Weight (centile) }\end{array}$} & $100 \%(49 / 49)$ & $100 \%(9 / 9)$ & & 1.000 \\
\hline & & & & \\
\hline$<25$ & $35 \%(14 / 41)$ & $0 / 9$ & & 0.047 \\
\hline \multirow{2}{*}{$\begin{array}{l}25-75 \\
>75\end{array}$} & $47.5 \%(19 / 41)$ & $44.4 \%(4 / 9)$ & & 1.000 \\
\hline \multirow{2}{*}{\multicolumn{5}{|c|}{ Height (centile) }} \\
\hline & & & & \\
\hline$<25$ & $33.3 \%(12 / 36)$ & $22.2 \%(2 / 9)$ & & 0.698 \\
\hline $25-75$ & $52.8 \%(19 / 36)$ & $33.3 \%(3 / 9)$ & & 0.727 \\
\hline$>75$ & $13.9 \%(5 / 36)$ & $44.5 \%(4 / 9)$ & & 0.063 \\
\hline \multicolumn{5}{|l|}{ OFC (centile) } \\
\hline \multirow{2}{*}{$\begin{array}{l}<50 \\
50-75\end{array}$} & $84.78 \%(39 / 46)$ & $44.44 \%(4 / 9)$ & & 0.017 \\
\hline & $13.04 \%(6 / 46)$ & $22.2 \%(2 / 9)$ & & 0.604 \\
\hline \multirow{2}{*}{$\begin{array}{l}75-98 \\
>98\end{array}$} & $2.17 \%(1 / 46)$ & $11.1 \%(1 / 9)$ & & 0.303 \\
\hline & $0 / 46$ & $22.2 \%(2 / 9)$ & & 0.024 \\
\hline Microcephaly & $54.35 \%(25 / 46)$ & $11.1 \%(1 / 9)$ & & 0.027 \\
\hline Occipital groove & $73.9 \%(17 / 23)$ & $50 \%(2 / 4)$ & & 0.558 \\
\hline Macrostomia & $100 \%(47 / 47)$ & $88.8 \%(8 / 9)$ & & 0.161 \\
\hline Protruding tongue & $70 \%(28 / 40)$ & $66.6 \%(6 / 9)$ & & 1.000 \\
\hline Wide-spaced teeth & $87.2 \%(34 / 39)$ & $62.5 \%(5 / 8)$ & & 0.123 \\
\hline Severe mental retardation & $100 \%(49 / 49)$ & $100 \%(9 / 9)$ & & 1.000 \\
\hline Seizures (presence) & $89.4 \%(42 / 47)$ & $44.4 \%(4 / 9)$ & & 0.006 \\
\hline Seizures (age of onset) (years) & $1^{7 / 12}$ & $6^{4 / 12}$ & 0.002 & \\
\hline (Range) & $2 / 12-4$ & $1^{6 / 12}-13$ & & \\
\hline Laughter outbursts & $95.8 \%(46 / 48)$ & $77.7 \%(7 / 9)$ & & 0.113 \\
\hline Ataxic gait & $93.1 \%(27 / 29)$ & $100 \%(8 / 8)$ & & 1.000 \\
\hline Hyperactivity & $94.3 \%(33 / 35)$ & $80 \%(4 / 5)$ & & 0.337 \\
\hline Capacity of communication & $73.1 \%(19 / 26)$ & $57 \%(3 / 4)$ & & 1.000 \\
\hline Sleep disturbance & $80.5 \%(29 / 36)$ & $71.4 \%(5 / 7)$ & & 0.624 \\
\hline Frequent drooling & $96.9 \%(32 / 33)$ & $87.5 \%(7 / 8)$ & & 0.356 \\
\hline Hyperphagia & $8.1 \%(4 / 49)$ & $33.3 \%(2 / 6)$ & & 0.123 \\
\hline Skin picking & - & $60 \%(3 / 5)$ & & - \\
\hline
\end{tabular}

$P:$ 1: Mann-Whitney's test.

2: Fisher's exact test.

Significant results $(P<0.05)$ are indicated in bold.

demeanor, with or without the presence of seizures, should be considered as minimal criteria for a SNURF-SNRPN exon 1 methylation assay test.

\section{Acknowledgements}

This work was supported by FAPESP (M.C.V. 99/10414-8), CEPID, and CNPq. We thank Roseli M Zanelato for technical assistance and Claudia IE de Castro for chromosome studies.

\section{References}

1 Angelman H: 'Puppet' children: a report on three cases. Develop Med Child Neurol 1965; 7: 681-688.

2 Christian SL, Robinson WPP, Huang B et al: Molecular characterization of two proximal deletion breakpoint regions in both Prader-Willi and Angelman syndrome patients. Am J Hum Genet 1995; 57: 40-48.

3 Christian SL, Fantes JA, Mewborn SK, Huang B, Ledbetter DH: Large genomic duplicons map to sites of instability in the Prader-Willi/Angelman syndrome chromosome region (15q11q13). Hum Mol Genet 1999; 8: 1025-1037. 
4 Amos-Landgraf JM, Ji Y, Gottlieb W et al: Chromosome breakage in the Prader-Willi and Angelman syndromes involves recombination between large, transcribed repeats at proximal and distal breakpoints. Am J Hum Genet 1999; 65: 370-386.

5 Wandstrat AE, Leana-Cox J, Jenkins L, Schwartz S: Molecular cytogenetic evidence for a common breakpoint in the largest inverted duplications of chromosome 15. Am J Hum Genet 1998; 62: $925-936$.

6 Roberts SE, Maggouta F, Thomas NS, Jacobs PA, Crolla JA: Molecular and fluorescence in situ hybridization characterization of the breakpoints in 46 large supernumerary marker 15 chromosomes reveals an unexpected level of complexity. Am J Hum Genet 2003; 73: 1061-1072.

7 Bolton PF, Dennis NR, Browne CE et al: The phenotypic manifestations of interstitial duplications of proximal $15 \mathrm{q}$, with special reference to the autistic spectrum disorders. Am J Med Genet 2001; 105: 675-685.

8 Ungaro P, Christian SL, Fantes JA et al: Molecular characterization of four cases of intrachromosomal triplication of chromosome 15q11-q14. J Med Genet 2001; 38: 26-34.

9 Roberts SE, Dennis NR, Browne CE et al: Characterization of interstitial duplications and triplications of chromosome 15q11q13. Hum Genet 2002; 110: 227-234.

10 Bottani A, Robinson WPP, DeLozier-Blanchet CD et al: Angelman Syndrome due to paternal uniparental disomy of chromosome 15: a milder phenotype? Am J Med Genet 1994; 51: 35-40.

11 Gillessen-Kaesbach G, Albrecht B, Passarge E, Horsthemke B: Further patient with Angelman syndrome due to paternal disomy of chromosome 15 and a milder phenotype. Am J Med Genet 1995; 56: 328-329.

12 Smith A, Marks R, Haan E, Dixon J, Trent RJ: Clinical features in four patients with Angelman syndrome resulting from paternal uniparental disomy. J Med Genet 1997; 34: 426-429.

13 Fridman C, Varela MC, Kok F, Diament A, Koiffmann CP: Paternal UPD 15: further genetic and clinical studies in four Angelman syndrome patients. Am J Med Genet 2000b; 92: 322-327.

14 Fridman C, Hosomi N, Varela MC, Souza AH, Fukai K, Koiffmann $\mathrm{CP}$ : Angelman syndrome associated with oculocutaneous albinism due to an intragenic deletion of the P Gene. Am J Med Genet 2003; 119A: 180-183.

15 Ohta T, Buiting K, Kokkonen $\mathrm{H}$ et al: Molecular mechanism of Angelman syndrome in two large families involves an imprinting mutation. Am J Hum Genet 1999; 64: 385-396.

16 Moncla A, Malzac P, Livet M-O et al: Angelman syndrome resulting from UBE3A mutations in 14 patients from eight families: clinical manifestations and genetic counselling. $J$ Med Genet 1999; 36: 554-560.

17 Lossie AC, Whitney MM, Amidon D et al: Distinct phenotypes distinguish the molecular classes of Angelman syndrome. J Med Genet 2001; 38: 834-845.

18 Fridman C, Varela MC, Valente K, Marques-Dias MJ, Koiffmann CP: Phenotypic and behavioral variability within Angelman syndrome group with UPD. Genet Mol Biol 2002; 25: 127-130.

19 Zeschnigk M, Lich C, Buiting K, Doerfler W, Horsthemke B: A single tube PCR test for the diagnosis of Angelman and PraderWilli syndrome based on allelic methylation differences at the SNRPN locus. Eur J Hum Genet 1997; 5: 94-98.

20 Mutirangura A, Jayakumar A, Sutcliffe JS et al: A complete YAC contig of the Prader-Willi/Angelman chromosome region (15q11-q13) and refined localization of the SNRPN gene. Genomics 1993; 18: 546-552.

21 Chai J-H, Locke DP, Greally JM et al: Identification of four highly conserved genes between breakpoint hotspots BP1 and BP2 of the Prader-Willi/Angelman syndromes deletion region that have undergone evolutionary transposition mediated by flanking duplicons. Am J Hum Genet 2003; 73: 898-925.

22 Rainier S, Chai JH, Tokarz D, Nicholls RD, Fink JK: NIPA1 gene mutations cause autosomal dominant hereditary spastic paraplegia (SPG6). Am J Hum Genet 2003; 73: 967-971.

23 Butler MG, Bittel DC, Kibiryeva N, Talebizadeh Z, Thompson T: Behavioral differences among subjects with Prader-Willi syndrome and type I or type II deletion and maternal disomy. Pediatrics 2004; 113: 565-573.

24 Fridman C, Koiffmann CP: Origin of uniparental disomy 15 in patients with Prader-Willi or Angelman syndrome. Am $\mathrm{J}$ Med Genet 2000a; 94: 249-253. 